\title{
Expert review on big data analytics implementation model in data-driven decision- making
}

\begin{abstract}
Data-driven decision-making can offer improved insights for information value and create new business opportunity. The purpose of this paper is to present the findings of expert opinion in verifying the influencing factors in big data analytics (BDA) implementation that are beneficial in developing the BDA implementation assessment model. The study was carried out by conducting face-to-face approach sessions with three academicians and four industry experts who have vast experience in big data research and its implementation. The findings from these exercises has confirmed and verified the content of the ten factors that include organization dimension (such as big data strategy, top management support, resource commitment, organizational relationship), people dimension (such as analytics skills, managerial skills and analytics culture) and technology dimension (includes data infrastructures, information processing and quality) were appropriate for the research model. It was described using descriptive analysis such as frequency, mean and standard deviation. Once the verification process is complete, the research model will be validated through survey in the future work.
\end{abstract}

Keyword: Expert review; Decision-making; Big data analytics implementation; Factors 